\title{
A New Automated Sample Transfer System for Instrumental Neutron Activation Analysis
}

\author{
S. S. Ismail \\ Atomic Institute, Vienna University of Technology, Stadionallee 2, 1020 Vienna, Austria \\ Correspondence should be addressed to S. S. Ismail, ismail@ati.ac.at \\ Received 1 December 2009; Accepted 25 January 2010 \\ Academic Editor: Peter Stockwell \\ Copyright () 2010 S. S. Ismail. This is an open access article distributed under the Creative Commons Attribution License, which \\ permits unrestricted use, distribution, and reproduction in any medium, provided the original work is properly cited. \\ A fully automated and fast pneumatic transport system for short-time activation analysis was recently developed. It is suitable \\ for small nuclear research reactors or laboratories that are using neutron generators and other neutron sources. It is equipped \\ with a programmable logic controller, software package, and 12 devices to facilitate optimal analytical procedures. $550 \mathrm{~ms}$ were \\ only necessary to transfer the irradiated capsule (diameter: $15 \mathrm{~mm}$, length: $50 \mathrm{~mm}$, weight: $4 \mathrm{gram}$ ) to the counting chamber at a \\ distance of 20 meters using pressurized air (4 bars) as a transport gas.
}

\section{Introduction}

A pneumatic transfer system (PTS) is required to facilitate the determination of very short-lived nuclides by instrumental neutron activation analysis (INAA) technique $[1,2]$. Semiautomatic PTS is not suitable for the determination of very short-lived nuclides (half lives $<1 \mathrm{~min}$ ) such as ${ }^{110} \mathrm{Ag},{ }^{80 \mathrm{~m}} \mathrm{Br},{ }^{38 \mathrm{~m}} \mathrm{Cl},{ }^{116 \mathrm{~m}} \mathrm{In},{ }^{20} \mathrm{~F},{ }^{179 \mathrm{~m}} \mathrm{Hf},{ }^{24 \mathrm{~m}} \mathrm{Na},{ }^{46 \mathrm{~m}} \mathrm{Sc},{ }^{77 \mathrm{~m}} \mathrm{Se}$, and ${ }^{207 \mathrm{~m}} \mathrm{~Pb}$. While automatic PTS performs the irradiation and measurements without manual manipulation between loading and counting procedures, these types of systems are fast, accurate, and comfortable to use in facilitating the determination of the above mentioned nuclides. However, they are not suitable for implementing sample exchangers to analyze large number of samples of various weights or matrices at optimal conditions. This is because the measurements are usually carried out at a fixed sampledetector distance.

Accurate measurements require the optimization of the input count rates of each measured sample, regardless of sample size, matrices or irradiation, and measuring techniques $[3,4]$. In comparison, the fully automated PTS, in addition to automatic irradiation measurement procedures, optimizes the sample-detector distances according to the count rates of the analyzed samples and the counting system. These systems are complex and expensive but provide accurate results. The first system using a digital gamma spectrometer to realize such features was published in 2001 [5]. This system optimizes not only the sample-detector distances (counting efficiency) according to the count rates (dead time) but also the shaping times (throughput/resolution). The work in this paper describes a fully-automatic rabbit system, which combines the potential of several systems and optimizes the sample-detector distance by setting the detector at a certain distance according to the expected count rates of the analyzed samples.

\section{Experimental}

2.1. Construction of the Sample Exchanger, Decay- and Depot Units. To facilitate the automatic analysis of about 30 samples a sample exchanger was constructed. The unit (Figure 1) consists of

(i) a polyamide tube (1) to store the samples, which are going to be analyzed;

(ii) a speed-fit adapter (2), to connect the polyamide tube to the main unit of the sample exchanger;

(iii) a separation device (3) which is powered pneumatically by a compact pneumatic cylinder (4); 


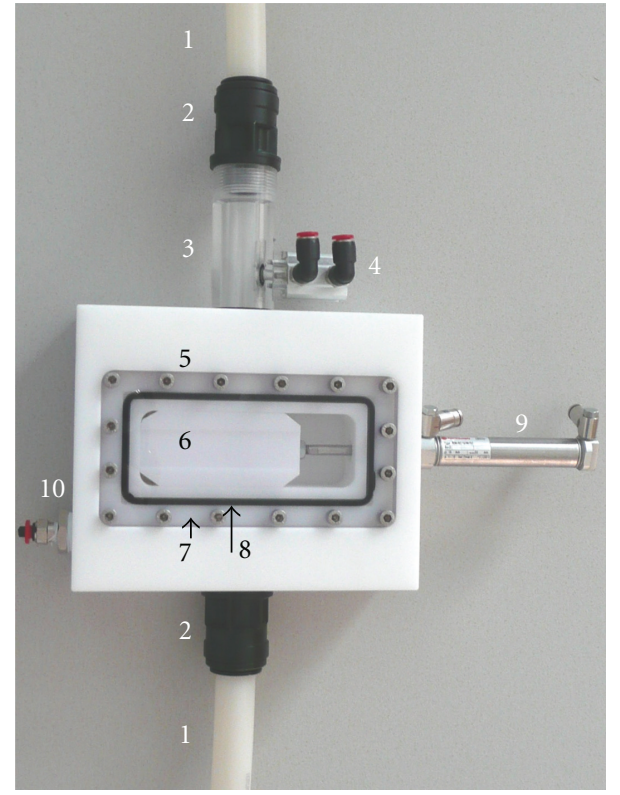

FIgURE 1: Components of the sample exchanger.

(iv) a sliding device (5) to introduce a sample into the loading unit; The main part of the unit (5) was made from polyoxymethylene (POM) while polyethylene (PE) was used to fabricate the moveable part (6); the front of the sliding device was fabricated from polycarbonate (PC) as a transparent window (7) to control the movements inside the unit;

(v) a sealing material (Nitrile Rubber; Butadiene Acrylonitrile) was used to surround the area (8) between the moveable part and the window in order to assure that the transport gas and contaminated friction particles do not escape from the unit,

(vi) a pneumatic cylinder (9) powers the unit;

(vii) an adapter (10) was installed to facilitate introducing the pressurized transport gas (air) for transferring the sample to the loading unit;

(viii) the sample exchanger is connected to the loading unit through an adapter (2b) and a polyamide tube (1b).

The same construction and materials were used to fabricate a decay and depot units.

2.2. Construction of the Sliding Devices, Loading and Separation Units. Figure 2(a) shows the construction of a tri directional sliding device (diverter). The moveable part (5) of the sliding device was fabricated mainly from polyethylene, while polyamide tubes $(19 / 22 \mathrm{~mm}$ ) were used inside this part. Two units were fabricated; one of them was implemented in front of the irradiation chamber, while the second was installed in front of the counting chamber. The units are powered pneumatically with multiposition pneumatic cylinders (6).

A bidirectional sliding device (Figure 2(b)) was constructed and integrated in the system. This unit was necessary for receiving the samples from the separation unit or from the decay station and directing them to the counting chamber. Each unit is airtight and fabricated from PA, PE, POM, and PC materials. Polyoxymethylene (POM) is a lightweight, low-friction thermoplastic material with good physical and processing properties. The main advantage of this material is its combination of strength, rigidity, and impact resistance.

Two loading units (Figure 2(c)) were constructed to receive samples manually (1) or from the sample exchangers (3). The units send the received samples to the irradiation position or the counting chamber through the middle adapter (2). The transport gas is connected to the unit through adapter-4. Each unit was constructed to be powered pneumatically by a pneumatic cylinder (6). The units are also equipped with a frame (7) to facilitate the installation in the main system.

A separation unit (Figure 2(d)) was constructed to receive the sample after irradiation through adapter- 2 and to direct the transport gas with the radioactive friction particles through adapter- 5 into the air filter unit. The unit sends the irradiated sample by clean transport gas (adapter-6) into the counting chamber through adapter-3. Additionally, the unit can forward the irradiated sample into a decay station through adapter-4. The units are airtight and fabricated from PE, POM, PC, and PA materials.

\subsection{Construction of the Irradiation and the Counting Cham-} bers. Figure 3 shows the components of the irradiation unit which accommodates the sample during the irradiation period. The unit was fabricated to be suitable for neutron sources such as neutron generators, microtrons, and so forth. The unit consists of two aluminum tubes (1 and 2) and two flanges ( 3 and 4 ). The two $\mathrm{Al}$ tubes have the dimensions of $17 / 20 \mathrm{~mm}$ and $24 / 28 \mathrm{~mm}$ and are arranged concentrically. The smaller tube is used for transportation of the sample capsule (outer diameter of $15.3 \mathrm{~mm}$ ), while the larger tube is used for the transport/expelling gas. Laser technique was used for welding the tubes to the flanges. Helium lack tests were performed to ensure that the unit is sealed. The metallic part of unit has a length of $80 \mathrm{~cm}$, while polyamide tubes (PA12) were used in the rest of the system. The irradiation unit is also equipped with an optical sensor (5) which is installed at a suitable position outside of the irradiation area.

A second irradiation unit was fabricated using the same construction but has a length of $300 \mathrm{~cm}$ to be installed inside a tank of a small research reactor (i.e., TRIGA Mark II).

A counting chamber (Figure 4) was fabricated to accommodate and center the irradiated sample in front of the detector during measurements. The unit is airtight and fabricated from POM and Plexiglas materials. To optimize the sample-detector distance, a method was developed to adjust the sample distance according to the activity of the analyzed samples $[5,6]$. The method in this work depends on a pneumatically powered moveable track which sets the detector at one of three positions according to the decision of the operator prior to measurement. The unit consists of three parts: a table (1) to set the detector on, a moveable truck (2) 


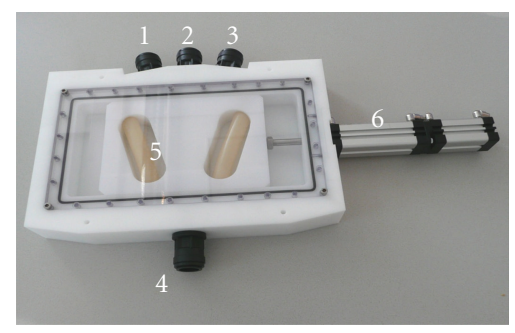

(a)

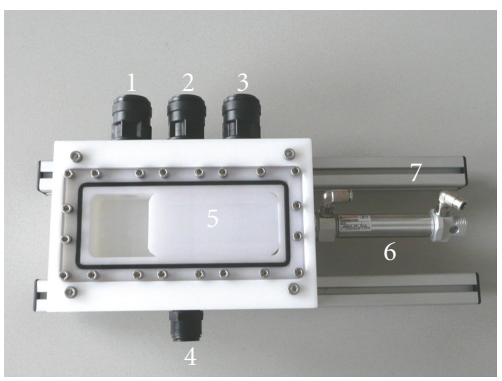

(c)

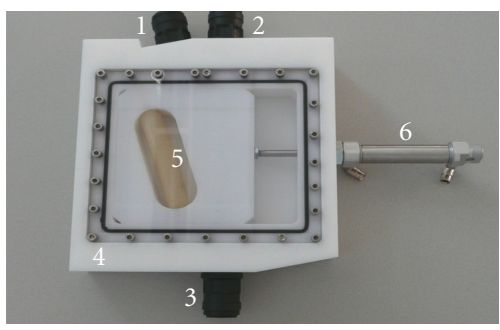

(b)

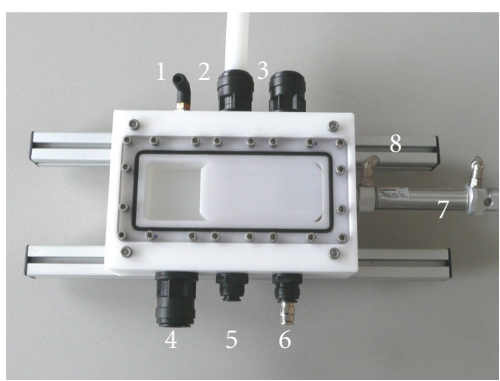

(d)

Figure 2: Components of the sliding devices ((a) and (b)) loading (c) and separation units (d).

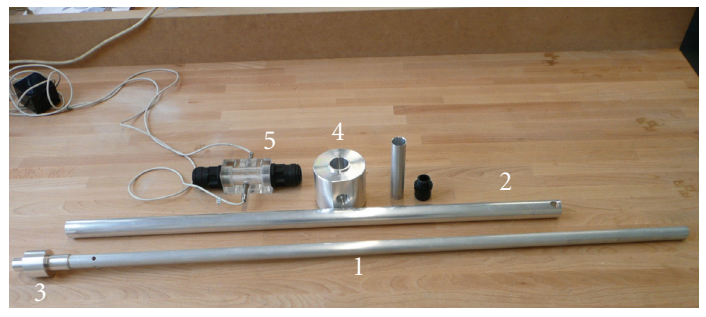

FIGURE 3: Components of the irradiation unit.

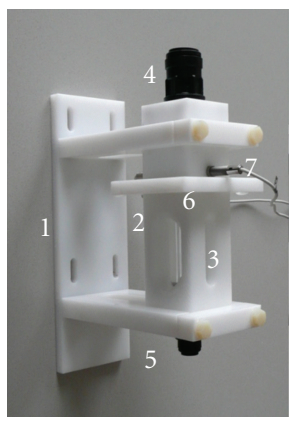

(a)

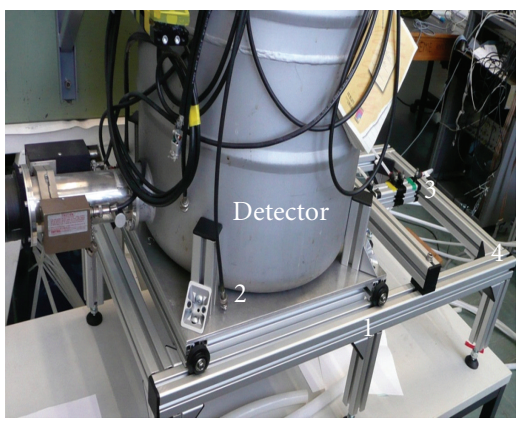

(b)
FIgure 4: Components of the counting units.

for moving the detector away from the measured sample, and a system for moving (3) the detector between three positions $(3,6$, and $9 \mathrm{~cm})$. This part consists of a flange mounted on the moveable truck of the detector and a multiposition pneumatic cylinder (4). The unit is powered pneumatically, with special arrangement to move the detector with suitable velocity.

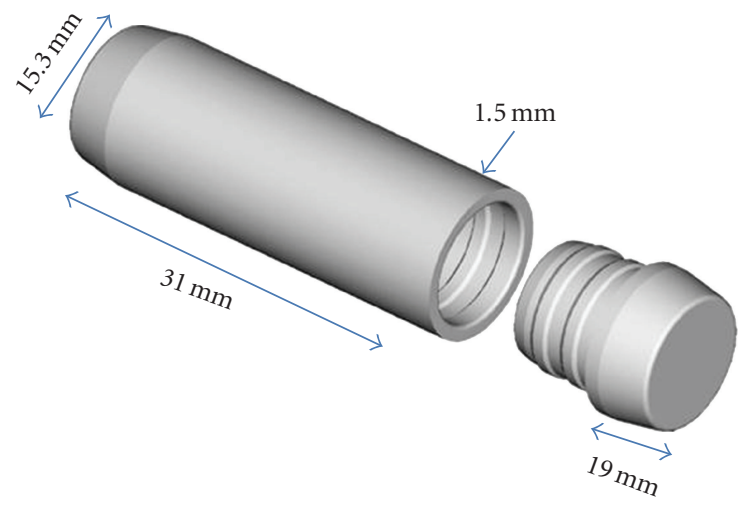

Figure 5: Dimensions of the irradiation capsule.

2.4. Construction of the Sample Capsule. A sample capsule with an inner volume of $3.5 \mathrm{~mL}$ and a total length of $50 \mathrm{~mm}$ was constructed to fit the requirements of the transport system. The outer diameter of the new capsule $(15.3 \mathrm{~mm})$ allows the use of commercial speed-fit adapters and polyamide tubes at an economical price. The inner diameter of $12.3 \mathrm{~mm}$ allows the introduction of small sample vials into the capsule. The capsule (Figure 5) was fabricated (by the help of a plastic company in Netherlands) from highdensity polyethylene (HDPE) with a push-in cap, avoiding the necessity to weld each capsule before use. The capsule was constructed to be used in an operating air pressure of 6 bars.

2.5. Construction of the Filter Units. To collect the waste air from the system, a small tank (CU) was fabricated from Plexiglas and two flanges (Figure 6). The tank was equipped with five nonreturn valves ( $\mathrm{x} 5$ ) and connected to two filter units. The filter unit consists of two filters; the first filter (F1) 


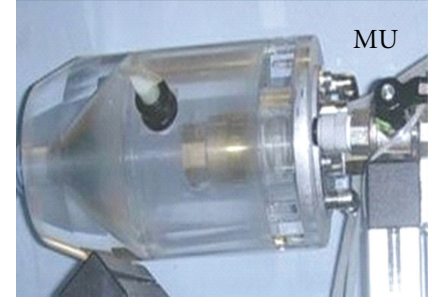

(a)

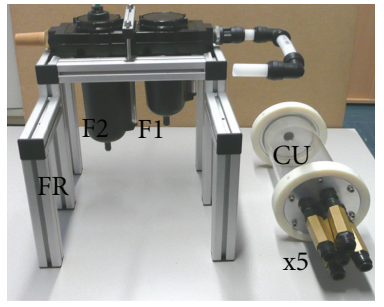

(b)
FIgure 6: Components of the air filter units.

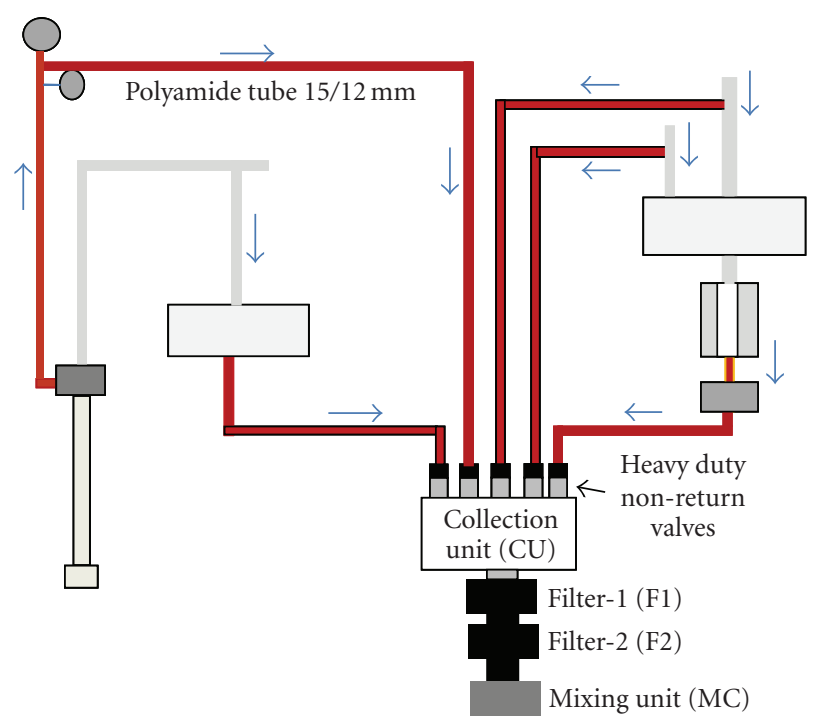

FIgURE 7: The main components of the waste air units.

is a prefilter for removing particles down to $5 \mu \mathrm{m}$ while the second filter (F2) cleans the waste air of particles down to $0.01 \mu \mathrm{m}$. After filtration, the waste air will be directed to a chamber (MU) to mix it with clean, pressurized air and then direct the resulting purified air to the main filter of the laboratory building. The MU chamber was fabricated from Plexiglas and equipped with a nonreturn valve and five adapters for feeding the clean, pressurized air. The filter units are installed and integrated in the system as shown in (Figure 7).

2.6. Pneumatic and Air Supplying Units. The required pressurized air ( 6 bars) is supplied using an air compressor with a main tank of $50 \mathrm{~L}$ (Figure 8). The main tank provides the pressurized gas to another two buffer tanks, each 30 liters, which are used for supplying 16 pneumatic cylinders and 22 valves with the necessary pressurized air at 2 and 4 bars, respectively. An air pressure sensor was installed at each tank to control the level of the air pressure. A series of adapters, pressure regulators, manometers and polyethylene tubes of different sizes $(6,12,10$, and $15 \mathrm{~mm})$ is integrated to optimize the operation of the pneumatic cylinders and valves. A polyamide tube $(\sim 19 \mathrm{~m})$ with a diameter of $17 / 22 \mathrm{~mm}$ was used to transport the sample unless at the irradiation chamber $(80 \mathrm{~cm})$. Therefore, the possible contamination through metallic tubes is largely avoided.
2.7. Control Unit and the Software Package. A control unit was fabricated to manage irradiation-measurement procedures (Figure 9). The unit is based on a $24 \mathrm{~V} / 4 \mathrm{~A}$ power supply (Siemens; LOGO-Power), programmable logic controller (PLC; NAiS-FP0), and a group of interfaces and adapters. The unit was constructed to facilitate the control of two valve islands (VM10, each 8 valves) through two interfaces (D-sub 25), and 6 external higher flow rate valves and six sensors through individual adapters.

A software package was developed to manage communication with the control unit, pneumatic devices, sensors, and the irradiation-counting procedures. The software is a Delphi Code, facilitating several functions through three main interfaces. The first interface is for communicating with the control unit for controlling and testing all sensors and valves manually. The second interface is for performing semiautomatic procedures with manual control of all included units or analytical steps. The third interface facilitates fullyautomatic operations and communication with a digital gamma spectrometer for starting the measurements.

\section{Results and Discussion}

The constructed system consists of three main parts. The first is composed of the chambers and sliding devices which facilitate the fully-automatic irradiation-counting procedures. The second comprises the pneumatic, air supply and waste air treatment units that facilitate the movement of the samples inside the system. The third is the software package and control unit for managing the automatic analytical procedures. The first part consists of 12 units, which are arranged in 4 groups to facilitate the complete fully-automatic movement of analyzed samples (Figure 10).

The first group (G1) consists of

(i) a sample exchanger and a loading unit; the sample exchanger was constructed so that the number of samples, which can be used for automatic analysis, is flexible, depending on the length of the polyamide tube (sample magazine); the materials used in fabrication are free of metallic components to avoid possible contamination before irradiation; it was constructed in a compact manner in order to be installed inside a small $\mathrm{Pb}$-shield for handling preirradiated samples; therefore, it was possible to implement three units of the same construction in the system as a sample exchanger (I), a decay station (XI) and a depot unit (IX);

(ii) the loading unit (II) was constructed to be powered by a pneumatic cylinder and one valve only; this was realized by constructing a new moveable middle part of the sliding device. The units were fabricated from nonmetallic components to minimize the contamination of the analyzed samples before irradiation;

(iii) an optical sensor (O1, infrared-880 nm, open collector, $50 \mathrm{KHz}$ ).

The irradiation group (G2) consists of 4 types of devices; a tri directional sliding device (III), an irradiation chamber (IV), 


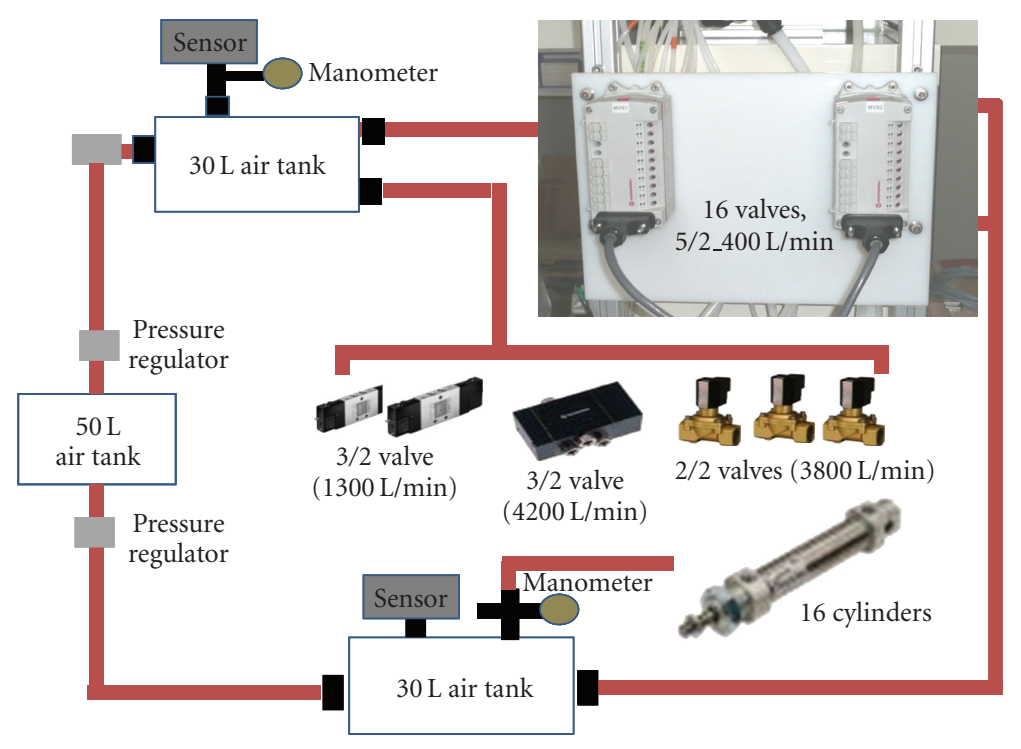

FIgURE 8: The main pneumatic components.

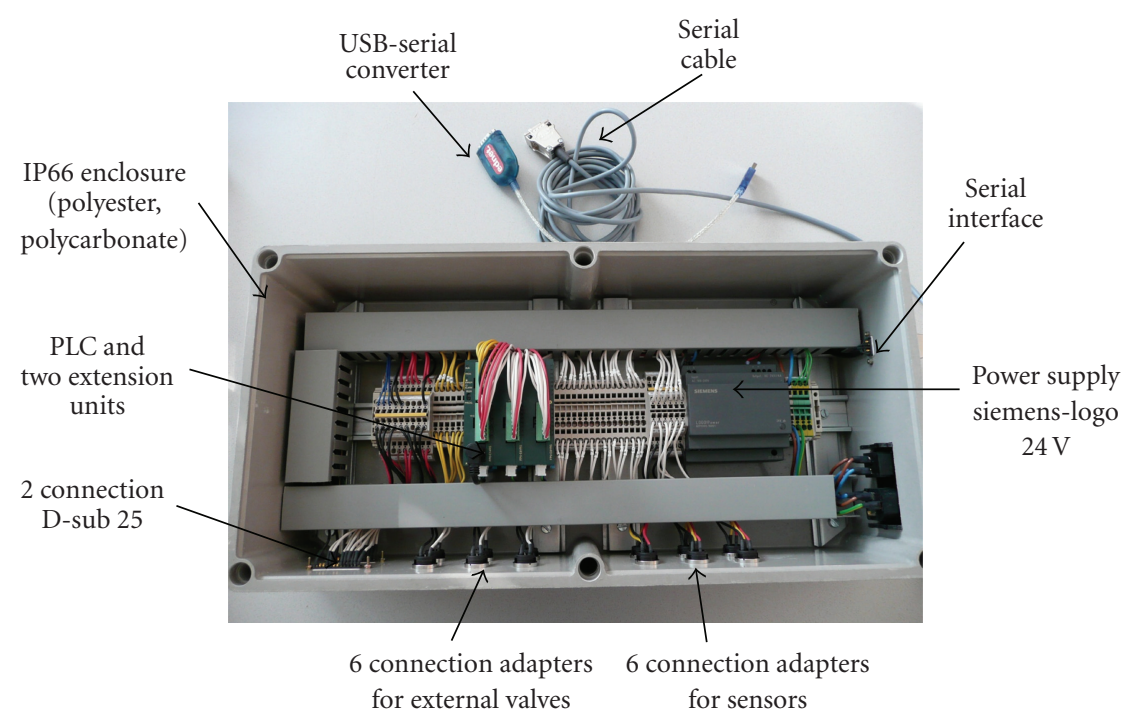

Figure 9: Components of control unit.

a separation unit $(\mathrm{V})$, and two optical sensors $(\mathrm{O} 2, \mathrm{O} 3)$. The construction of the $\mathrm{G} 2$ provides several advantages:

(i) the sliding device facilitates the introduction of the irradiation samples directly into the counting chamber for fast transport time and enables the measurement of very short-lived nuclides without any delay;

(ii) alternatively, it can direct the sample to the separation unit; and then send it to the counting chamber with clean transport gas; this step is necessary to avoid contamination of the counting chamber; it is powered by one pneumatic cylinder only in order to reduce the number of pneumatic devices in the system as well as to avoid the implementation of several complex, multipositional pneumatic cylinders; (iii) the implementation of two optical sensors $(\mathrm{O} 2, \mathrm{O} 3)$ enables automatic control and movement of the sliding devices at the correct time, as well as starting the counter for the irradiation period;

(iv) the fabrication of the irradiation chamber from an Aluminum alloy (ALMg Si)makes the manipulation, service and disposal of this unit simple; after thermal neutron activation, Al produces a short-lived radioactive nuclide $\left({ }^{28} \mathrm{Al}\right)$ with a half-life of 134 second. Therefore, $\mathrm{Al}$ is a suitable material for the fabrication of such units.

The third group (G3) consists of 6 units: two sliding devices (VI, VII), a counting chamber (VIII), an optical sensor (O4), a depot unit (IX), and a pneumatic track for moving a detector. G3 enables the introduction of the irradiated 


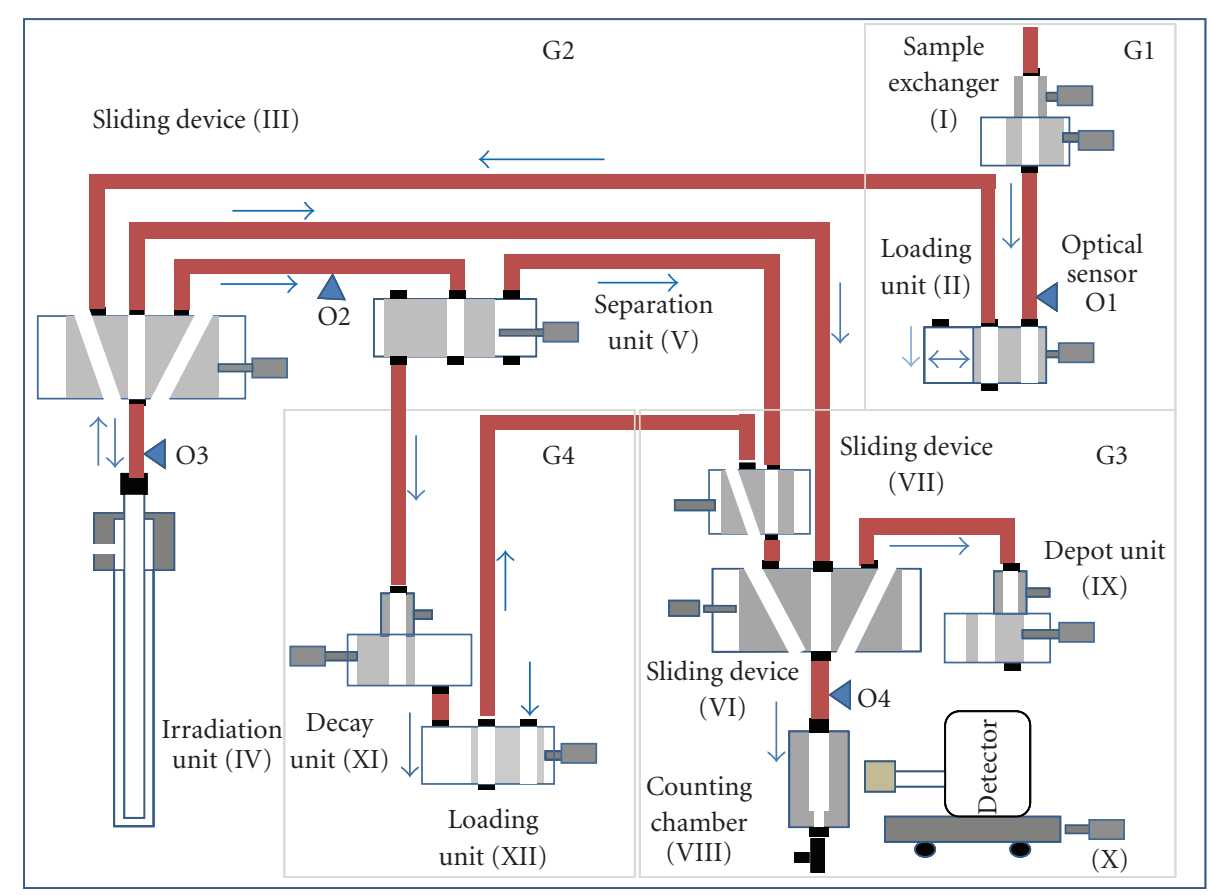

FIGURE 10: Arrangement of the irradiation-counting units.

samples into the counting chamber from three different sources, and it also sends the sample to the depot unit after measurement. Additionally, G3 is equipped with an optical sensor for automatically starting the measurements. The main advantage of the detector truck is that it can pneumatically set the detector at one of three positions (3, 6 , and $9 \mathrm{~cm}$ ) from the analyzed samples, thereby optimizing count rates. Furthermore, the construction facilitates cyclic activation by directing the sample to the irradiation unit after measurement.

The fourth group (G4) consists of a sample exchanger (XI) which acts as a decay station and a loading unit (XII) for automatically feeding the preirradiated samples into the counting chamber. This group possesses two advantages. The first is the ability to irradiate the sample and then wait for a suitable time for the measurements to optimize the analytical conditions (i.e., background) according to the half-lives of the investigated nuclides. The second is the possibility to use the unit as a standalone system for automatically analyzing preirradiated samples. This arrangement allows the new system to be used during the working hours for short-time activation analysis and overnight for measuring long-lived nuclides.

The second part of the system is the pneumatics, valves, and air supplying units that operate all devices and the movement of the samples inside the system. There are 10 units powered pneumatically using 16 pneumatic cylinders. The technical specifications of each cylinder depend on the duty and characteristics of each unit, since the precise operation and timing of the movement of the cylinders are very important for safe and automatic operations. Any deviation or delay could stop the operation or damage the irradiated sample inside the system. Therefore, some of these cylinders are operated at different air pressures (2 and 4 bars) or their movements are controlled by special adapters. Additionally, operating the cylinders or the sample inside the system required 22 solenoid valves, 16 of them with a flow rates of $4001 / \mathrm{min}$ and 6 with higher flow rates (4200, 3800 and $13001 / \mathrm{min})$. The operation time of each valve was controlled by software.

The third part of the system is the control unit and the software package to manage optimal operation and the analytical procedures. Figures 11, 12, and 13 show the interfaces of semiautomatic operations and controlling each unit and valve. The interfaces were developed to realize several operations and advantages:

(i) it allows the operating conditions of each valve involved in each step (i.e., operating time, delay time) to be optimized; this facility helps to adjust the conditions according to the distance between the irradiation and counting chambers without manipulating the source code of the software;

(ii) it allows the optical sensor to control the process; The sensors have two functions. The first is to guarantee that the sample has passed a certain point in the system; if that is not the case, the total process will be interrupted. The second is to start specific processes, such as irradiation time, loading a new sample, and starting the measurements. When using the sensors, the procedure is controlled by a time period (timeout) which is given to interrupt the process if the sample does not pass the point; 


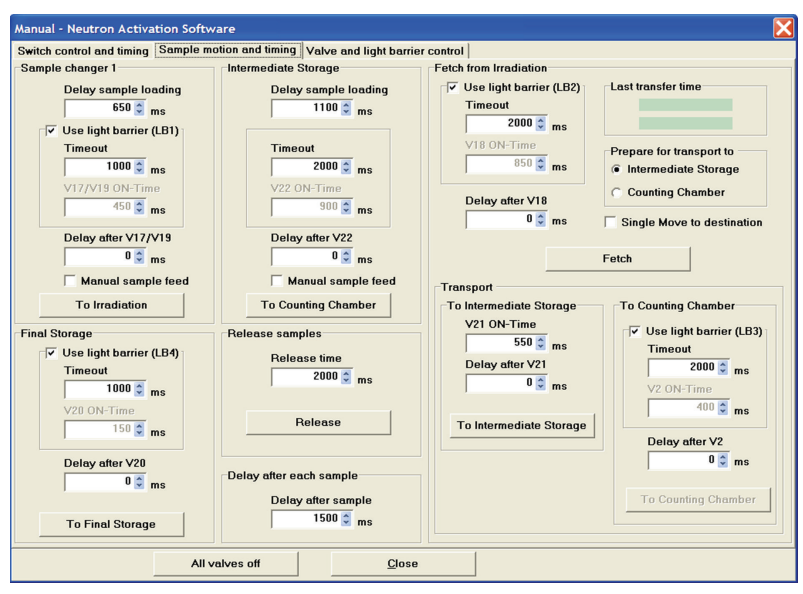

FIGURE 11: Sample motion and timing interface of the new software package.

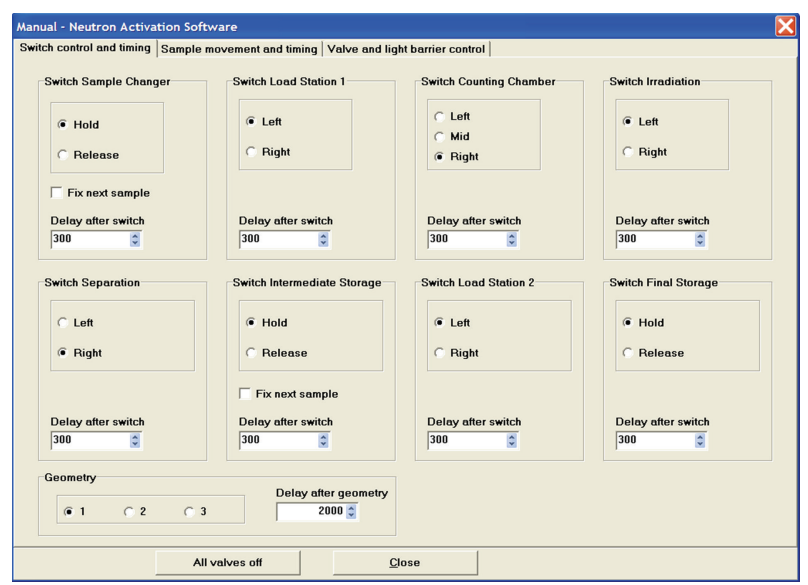

FIgURE 12: The control and timing interface of the new software package.

(iii) it allows semiautomatic operations for moving the sample between the units (i.e., loading, irradiation, counting, and depot);

(iv) it is used to determine if the sample, after irradiation, will be directed to the counting chamber for fast transfer time or to the separation unit.

(v) it provides information about the transfer time from two sources, the timer of the PC and PLC (control) unit, using its internal clock.

Figure 14 shows the main interface of the software package. It was developed to realize several operations such as

(i) documentation of (part-1) the necessary information for the archive of the analysis as well as the navigation to other interfaces, tools, and files;

(ii) preparation of the analytical conditions for measuring up to 20 spectra at different counting times and sample detector distances (part-2) following each irradiation; the software also allows the measurement

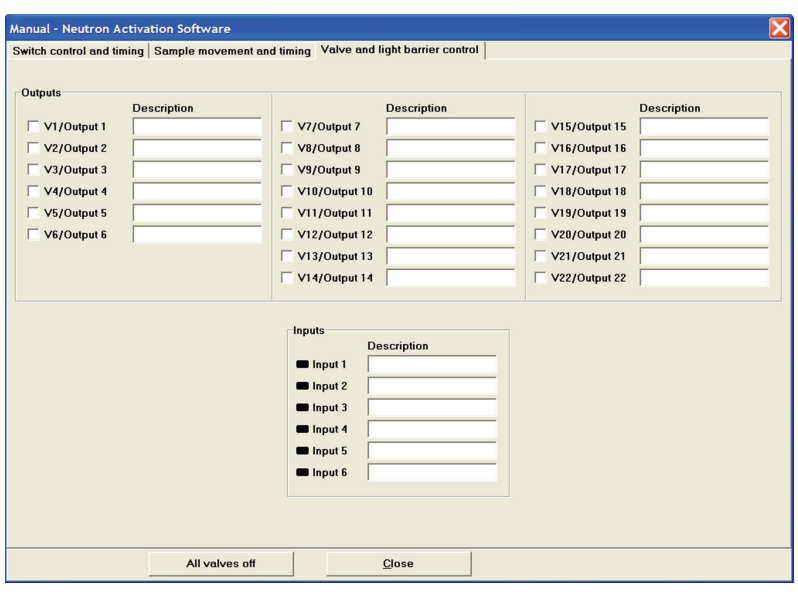

FIGURE 13: Valves and sensors interface.

of up to 999 spectra at a certain sample-detector distance and counting time;

(iii) deciding between one or more of three operation modes; irradiation, measurement, irradiation/measurements, and manual or automatic loading (part-3); if the samples should only be irradiated, then the samples will be transferred to the decay unit after irradiation. If the sample will only be measured, then it will be treated as a preirradiated sample and transferred from the decay station into the counting chamber;

(iv) setting the analytical conditions for all samples that should be automatically analyzed (part-4) with a minimum effort; each analyzed sample has its interface; therefore it is possible to define the irradiation, counting, and control conditions of each analyzed sample individually; this technique gives the operator the capability for a dynamic analysis; the operator can irradiate and measure an unirradiated sample and then measure a preirradiated sample waiting in the decay station;

(v) providing online information for the spectrum being measured, indicating the sample number, the sequence, and measuring time (part-5); additionally, it indicates the hardware devices which are involved, such as optical sensors (LB1-LB4), the spectrometer (MCA), and the pressure sensors (P1, P2);

(vi) setting up the detector at the optimal sample-detector distance prior to the measurements to optimize the count rates depends on the matrix of the analyzed sample and the irradiation-counting conditions.

The transfer time is the real test for the correct construction and optimization of the pneumatic components. The result obtained (30 replicates) for measuring the transfer time over a distance of $20 \mathrm{~m}$ (the direct distance between the irradiation unit and the counting chamber) was found to be $0.55 \pm 0.01 \mathrm{~s}$ for a 4 -gram sample at air pressure of 4 bars (Figure 15), while the transfer time was $0.79 \pm 0.03 \mathrm{~s}$ at an air pressure of 2 bars. 


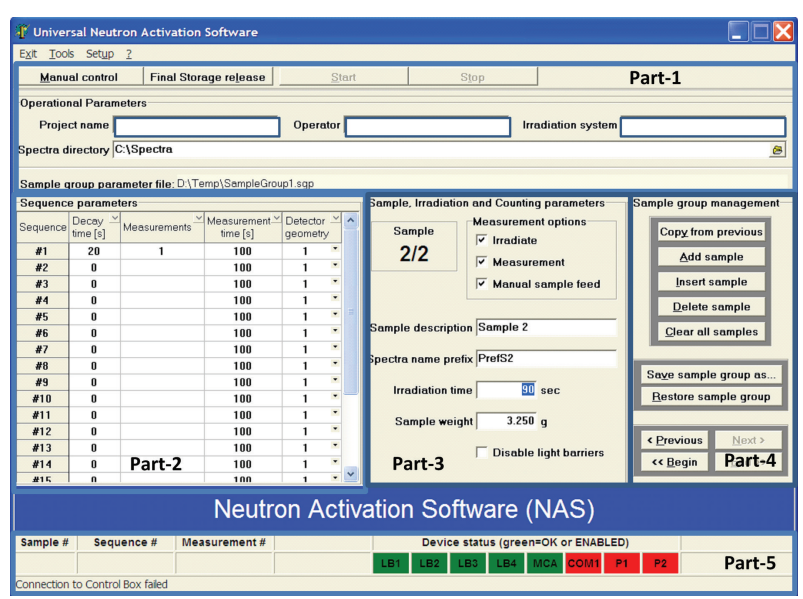

FIGURE 14: The main interface of the new software package.

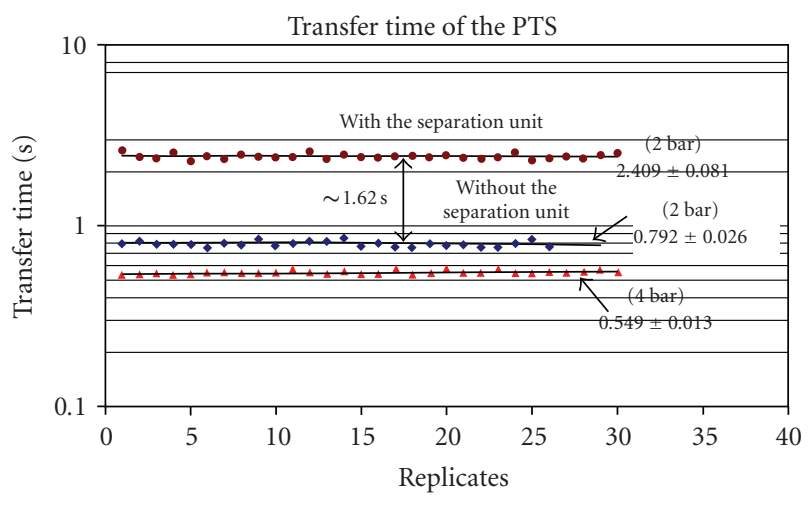

FIgURE 15: The transfer time at different conditions.

A series of reference materials was prepared for shorttime activation: Coal Fly Ash, CFA-1633b; Coal-1632; City Waste Incineration Ash, BCR-176; Sewage Sludge, BCR146; Lake Sediment, IAEA SL-1; Lake Sediment, IAEA SL3; Granite, SARM-1; and SARM-5. Four replicates of each material $(100 \mathrm{mg})$ were prepared for short-time activation analysis [4]. The samples were irradiated for 10, 40, 160, and $640 \mathrm{~s}$, respectively, at the TRIGA Mark-II reactor (Atomic Institute) using the fast rabbit irradiation system at a neutron flux of $2.7 \times 10^{12} \mathrm{n} \cdot \mathrm{cm}^{-2} \cdot \mathrm{s}^{-1}$. After each irradiation, the samples were counted for $10,40,120$, and 600 s (endcap $14 \% \mathrm{HPGe}$ detector) at a fixed sample-detector distance of $9 \mathrm{~cm}$. In general, after comparing all results obtained, the analyzed reference materials can be classified according to the activity after irradiation in two groups. The first group contains materials, such as CFA, SL-1, BCR-176, and SARM1 , which are producing high-count rates, so care should be taken in analyzing such materials, at short sample-detector distances. The second group includes materials, such as Coal, SL-3, BCR-146, and SARM-5, which are producing low-count rates, thus allowing analysis of these samples directly after the end of irradiation at short sample- detector distances (i.e., $3 \mathrm{~cm}$ ). The difference between input count rate (ICR) at certain irradiation and delay times simply indicates the effect of the matrix on the produced activity.
It was indicated that a matrix, such as CFA, produces an ICR of factors 5 and 15 higher than BCR-176 and SL-3, respectively. Thus, it is possible to calculate the ICR of each matrix at any analytical condition (irradiation, delay times). These results help to optimize analytical conditions, such as sample weight, irradiation and delay time as well as counting geometry and the use of the decay station to keep the input count rates of any analyzed material within the limit of 200 kcps.

\section{Conclusion}

The new system provides a fast and fully-automatic shorttime activation analysis. The construction guarantees accurate, safe, dynamic, and modern operations. The construction and implementation of an automatic separation unit, decay station, a second loading unit, and a moveable arrangement for the detector provide dynamic analysis at optimal conditions.

\section{Acknowledgments}

The author would like to thank IAEA for ordering a similar system for a nuclear analytical laboratory in Cuba. The author would like to thank Mr. W. Klikovich (Atomic Institute) for his kind help during this work.

\section{References}

[1] S. J. Parry, Activation Spectrometry in Chemical Analysis, John Wiley \& Sons, London, UK, 1991.

[2] Y.-S. Chung, S.-H. Kim, J.-H. Moon, et al., "New pneumatic transfer systems for neutron activation analysis at the HANARO research reactor," Journal of Radioanalytical and Nuclear Chemistry, vol. 278, no. 3, pp. 707-712, 2008.

[3] S. S. Ismail, "Quality of high count rate high resolution shorttime activation analysis," Journal of Radioanalytical and Nuclear Chemistry, vol. 213, no. 4, pp. 291-310, 1996.

[4] S. S. Ismail, "High counting efficiency compton suppression system for automatic-INAA," Instrumentation Science \& Technology, vol. 35, no. 3, pp. 307-322, 2007.

[5] S. S. Ismail, K. Brezovits, and W. Klikovich, "Dynamic irradiation counting systems for fast, short-time activation analysis using a fully automatic moveable counting chamber and a digital spectrometer," Instrumentation Science \& Technology, vol. 29, no. 4, pp. 255-266, 2001.

[6] S. S. Ismail and W. Klikovich, "Low cost fully-automatic counting system for INAA," Egyptian Journal of Chemistry, vol. 51, no. 1, pp. 29-42, 2008. 


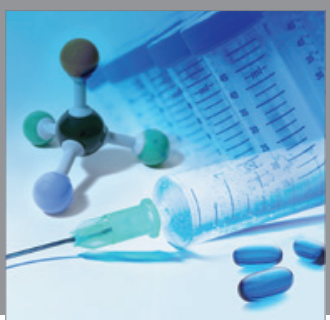

International Journal of

Medicinal Chemistry

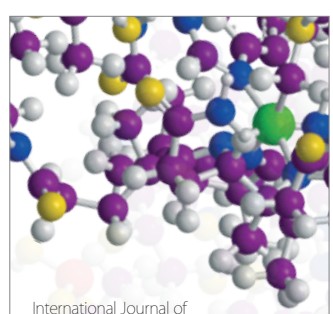

Carbohydrate Chemistry

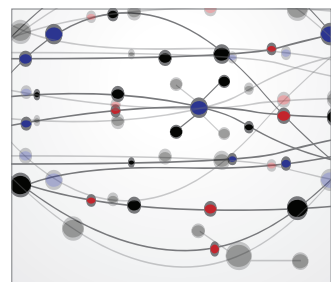

The Scientific World Journal
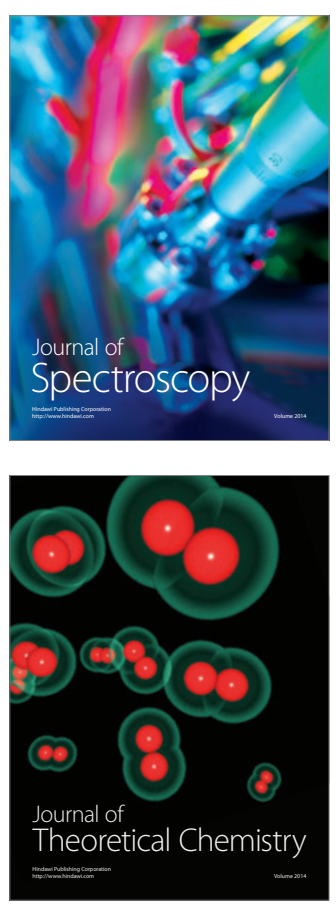
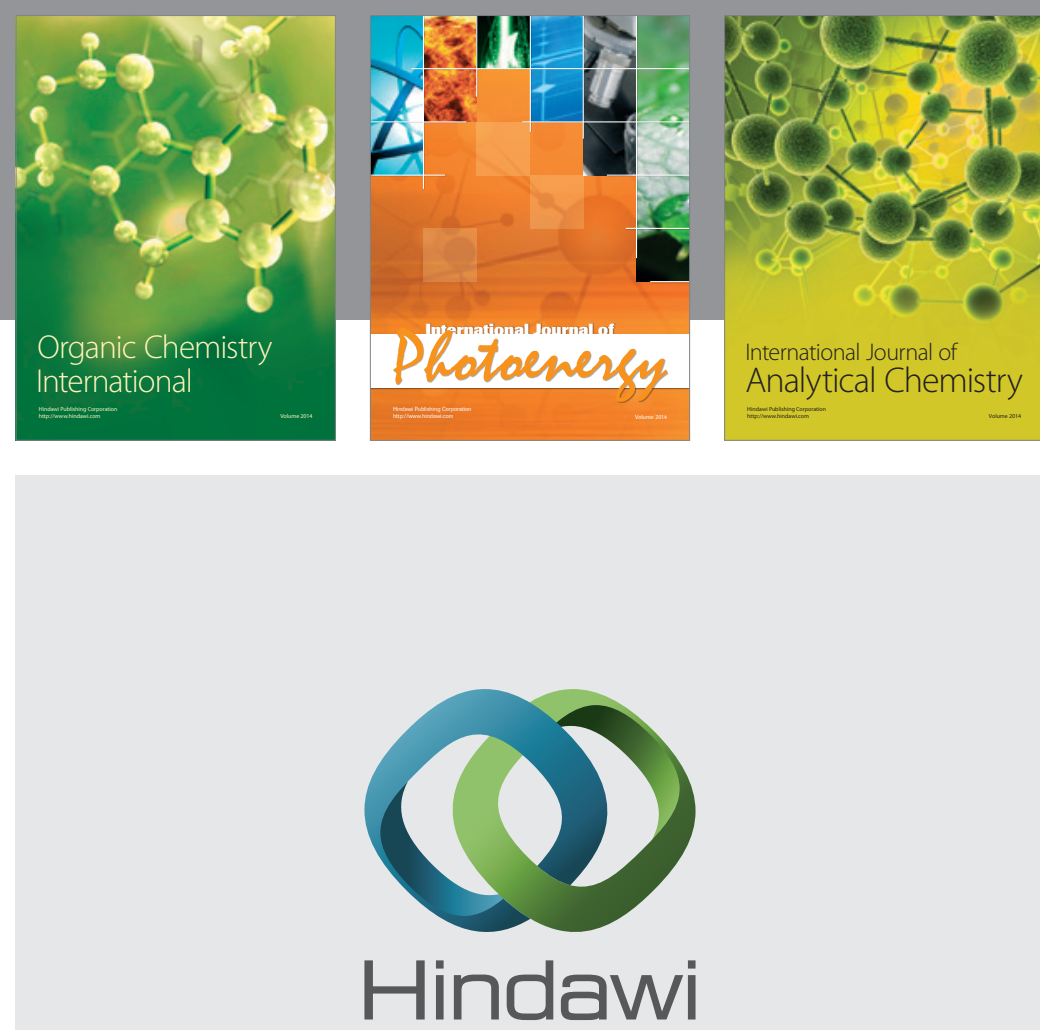

Submit your manuscripts at

http://www.hindawi.com
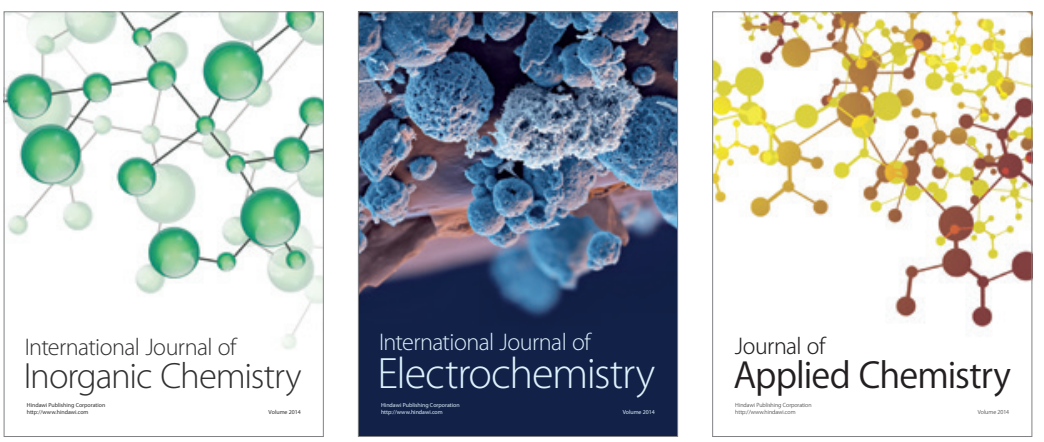

Journal of

Applied Chemistry
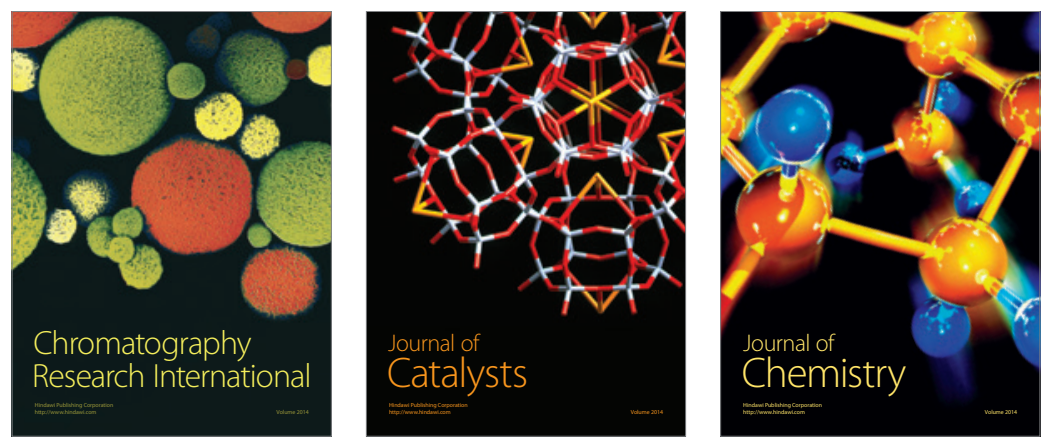
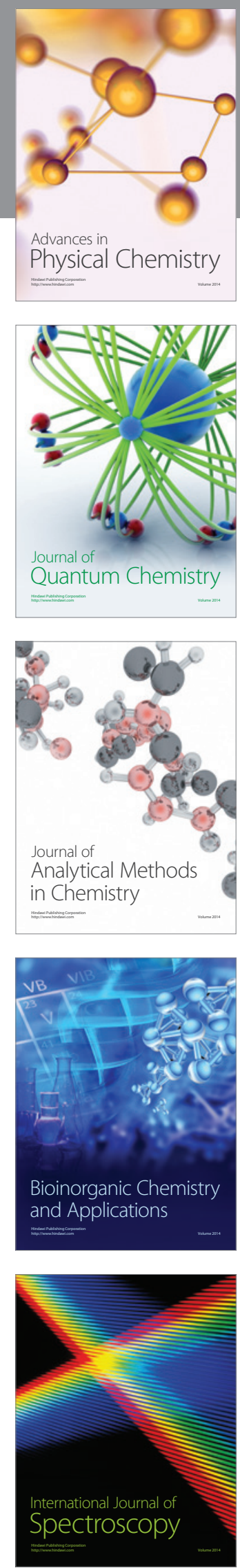